\title{
Publisher Correction: Reciprocal regulation of cellular mechanics and metabolism
}

\section{Tom M. J. Evers, Liam J. Holt (D), Simon Alberti and Alireza Mashaghi (D)}

Correction to: Nature Metabolism https://doi.org/10.1038/s42255-021-00384-w, published online 19 April 2021.

In the version of the article originally published, Fig. 2 contained scientific errors and unclear graphical rendering. In Fig. 2a, the misalignment of the tubulin dimers and the arrows pointing to the ends of the actin filaments have been corrected; the rendering of actin has been revised; and space has been added between intermediate-filament subunits for clarity. In Fig. $2 c$, errors in the numbers and locations of phosphate groups in $\mathrm{PIP}_{2}$ and PIP ${ }_{3}$ have been corrected. In Fig. 2d, Rho, ROCK, MLCK and MLC have been separated by arrows, and the rendering of the interaction between the ECM and integrin has been revised. The errors have been corrected in the HTML and PDF versions of the article.

a

Original

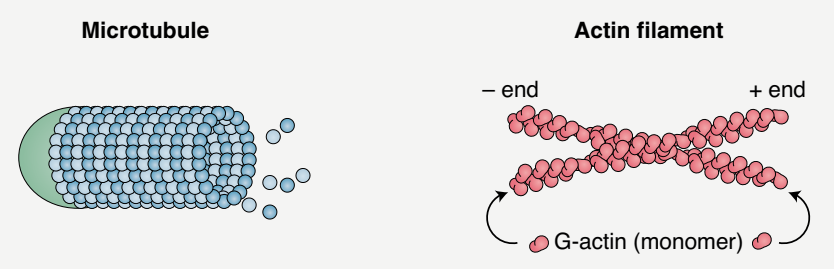

Intermediate filament

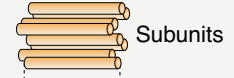

$\bigcirc$ One unit

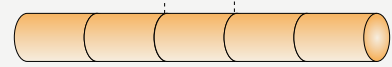

b

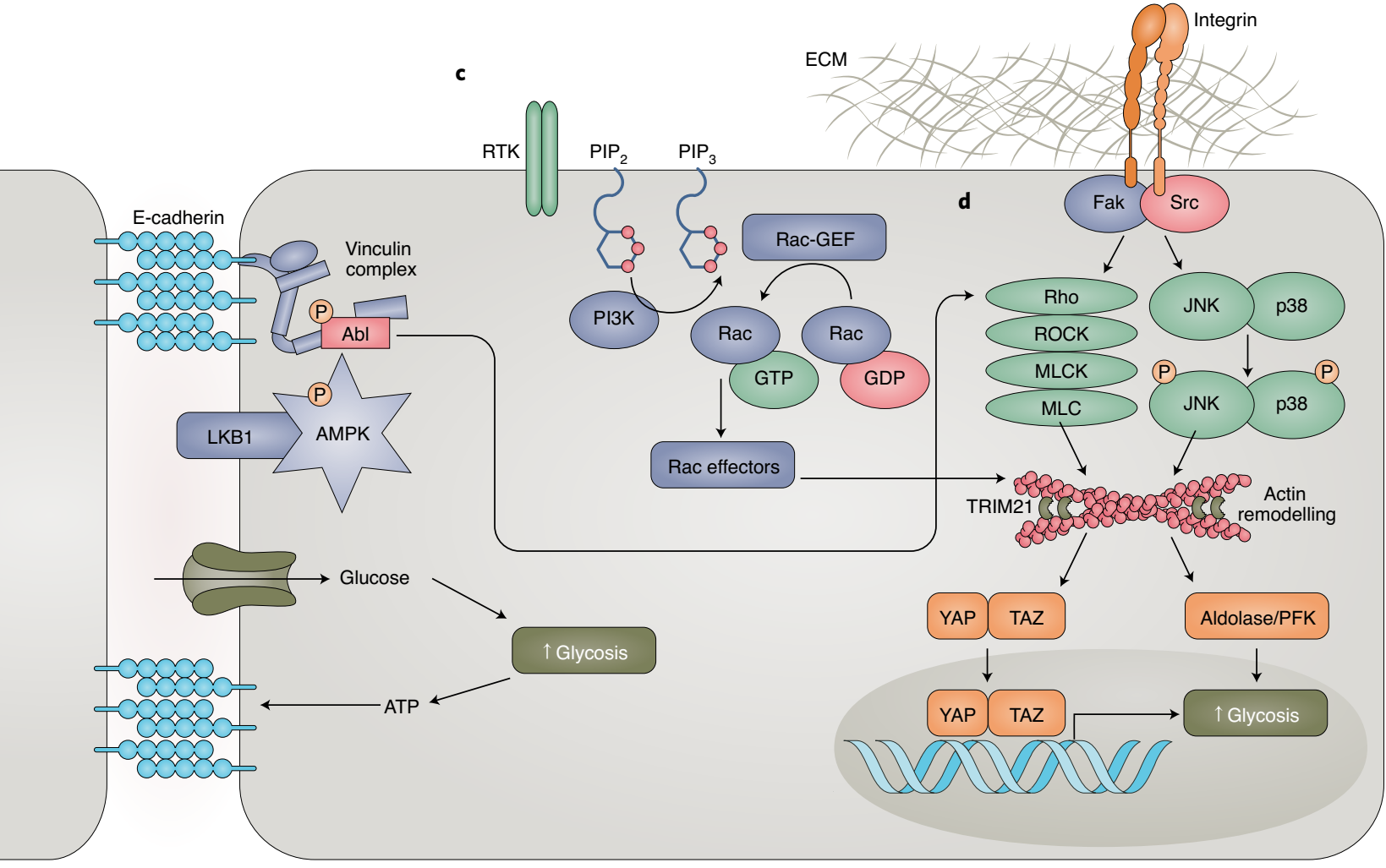




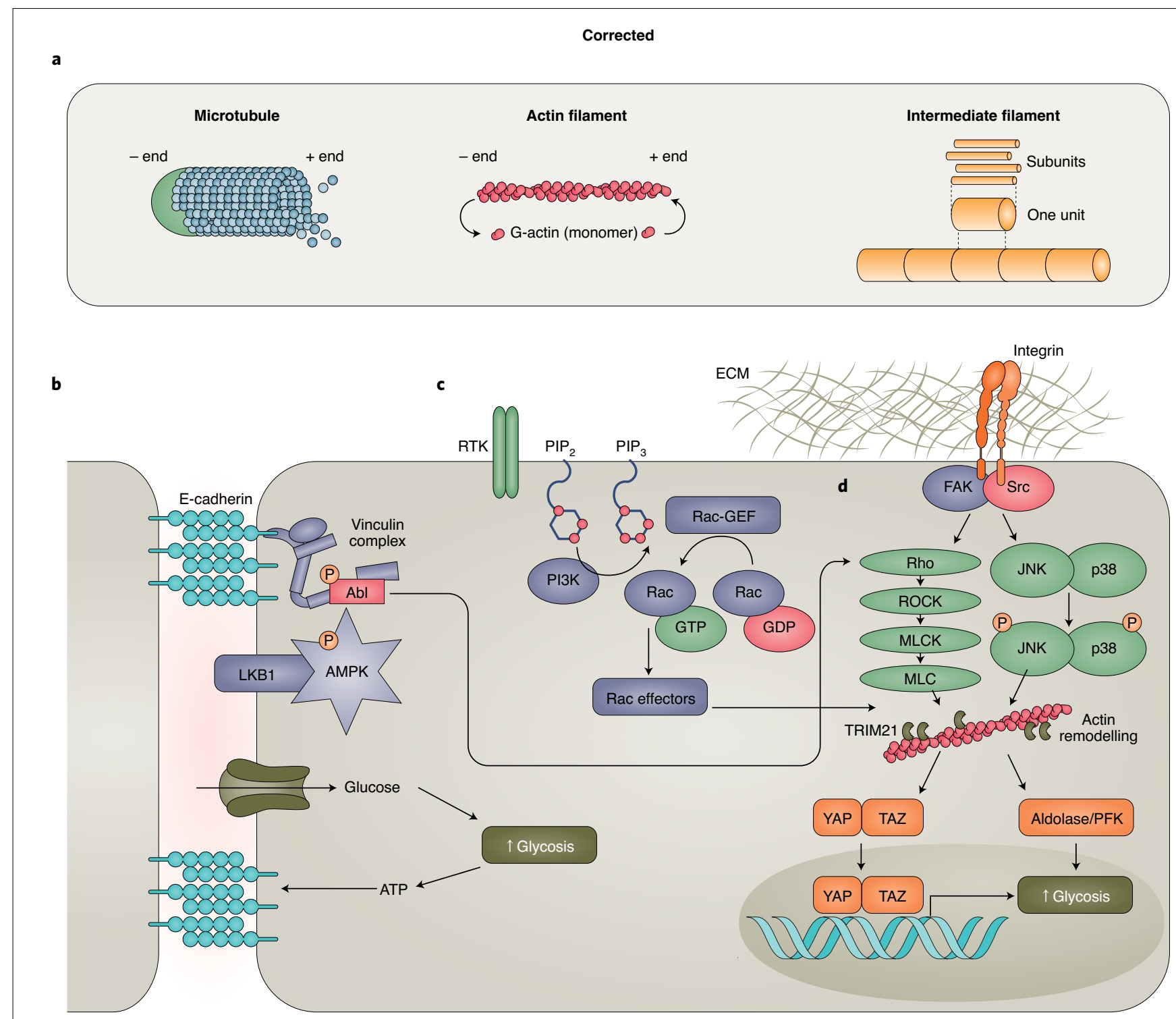

Fig. 2 | Original and corrected.

Published online: 9 June 2021

https://doi.org/10.1038/s42255-021-00423-6

๑) Springer Nature Limited 2021 